\title{
\begin{tabular}{l|l} 
Mitraries & DSpace@MIT
\end{tabular}
}

\author{
MIT Open Access Articles
}

\section{Tunable Infrared Emission From Printed Colloidal Quantum Dot/Polymer Composite Films on Flexible Substrates}

The MIT Faculty has made this article openly available. Please share how this access benefits you. Your story matters.

Citation: Panzer, M.J. et al. “Tunable Infrared Emission From Printed Colloidal Quantum Dot/ Polymer Composite Films on Flexible Substrates." Display Technology, Journal of 6.3 (2010): 90-93. (c) 2010 IEEE

As Published: http://dx.doi.org/10.1109/jdt.2009.2034276

Publisher: Institute of Electrical and Electronics Engineers

Persistent URL: http://hdl.handle.net/1721.1/61690

Version: Final published version: final published article, as it appeared in a journal, conference proceedings, or other formally published context

Terms of Use: Article is made available in accordance with the publisher's policy and may be subject to US copyright law. Please refer to the publisher's site for terms of use. 


\title{
Tunable Infrared Emission From Printed Colloidal Quantum Dot/Polymer Composite Films on Flexible Substrates
}

\author{
Matthew J. Panzer, Vanessa Wood, Scott M. Geyer, Moungi G. Bawendi, and Vladimir Bulović
}

\begin{abstract}
A simple and robust device structure for a flexible, multicolor infrared (IR) display is described. The display operates by optical downconversion of AC-driven blue phosphor electroluminescence using different-sized, IR-emitting colloidal quantum dots. Deposition of the IR emissive layer via inkjet printing facilitates side-by-side multicolor pixel definition with low material losses.
\end{abstract}

Index Terms-Infrared displays, flexible structures, quantum dots (QDs), ink jet printing.

\section{INTRODUCTION}

$\mathbf{T}$ HE development of tunable, electrically driven infrared (IR) emissive devices is currently being explored for possible applications in telecommunications and secure information display. IR-emitting materials under recent investigation have included lanthanide-doped inorganics [1], organic donor-acceptor molecules [2], [3], and conducting polymers or small molecule organic semiconductors mixed with organic dyes [4] or inorganic semiconductor nanocrystals [5], [6]. In order to obtain electrically activated IR emission, previous efforts have focused on embedding the emissive layer into a diode architecture, leading to current-driven devices that typically exhibit low external quantum efficiencies $(\sim 0.5 \%)$ at emission wavelengths beyond $1 \mu \mathrm{m}$ [4]-[6]. The diode approach also requires careful alignment of the energy levels between the electron/hole transport layers and the emissive material to obtain effective injection of both carrier types into the emissive layer, balanced charge recombination, and, in the case of organic transport layers, efficient excitonic energy transfer [7], [8].

In this report, we present an alternative architecture for obtaining large area IR emission, tunable across the 1-1.6 $\mu \mathrm{m}$ range, which does not rely on direct carrier injection. Instead, a

\footnotetext{
Manuscript received June 19, 2009; revised September 27, 2009. Current version published February 10, 2010. This work made use of MRSEC Shared Experimental Facilities at MIT, supported by the National Science Foundation under Award DMR-08-19762. This work was supported by the Army Research Office through the MIT Institute for Soldier Nanotechnologies (W911NF-07-D0004), the DARPA MIT-OSU-HP Focus Center on Non-Lithographic Technologies for MEMS/NEMS, a Presidential Early Career Award for Scientists and Engineers, and a NDSEG Fellowship.

M. J. Panzer, V. Wood, and V. Bulović are with the Electrical Engineering and Computer Science Department, Massachusetts Institute of Technology, Cambridge, MA 02139 USA (e-mail: mpanzer@mit.edu; vwood@mit.edu; bulovic@mit.edu).

S. M. Geyer and M. G. Bawendi are with the Chemistry Department, Massachusetts Institute of Technology, Cambridge, MA 02139 USA (e-mail: sgeyer@mit.edu; mgb@mit.edu).

Digital Object Identifier 10.1109/JDT.2009.2034276
}

high frequency, alternating current $(\mathrm{AC})$ voltage signal is used to excite an electroluminescent powder phosphor, generating blue photons that are absorbed and subsequently re-emitted at longer wavelengths by an adjacent IR emissive layer. The operating principle of these structures is, therefore, optical downconversion, not direct electrical excitation of the emissive material. An advantage of this approach is that AC-driven powder and thin film electroluminescence is already a mature field, with commercial products for visible displays in use today [9]. To adapt an established technology for visible light emission into a tunable IR display, we insert an IR-emissive layer in front of the phosphor layer such that the blue, AC-driven phosphor electroluminescence can be absorbed and then re-emitted via photoluminescence of the IR material, viewable through the substrate.

The IR-emissive material set used in this study is a series of colloidally synthesized $\mathrm{PbS} / \mathrm{CdS}$ core/shell quantum dots (QDs). Alternatively, any suitable IR emitter could be used, provided that it absorbs blue light and is chemically compatible with the overlying phosphor layer. QDs are particularly attractive as IR emitters because they can be synthesized in large quantities in solution, and their peak emission wavelength can be tuned based on their size due to the effects of quantum confinement. A schematic cross-section of an individual core/shell QD is depicted in Fig. 1(a). Atomic force microscopy of films of close-packed $\mathrm{PbS} / \mathrm{CdS}$ QDs is used to measure a typical QD diameter of $8 \mathrm{~nm}$. The organic ligands that coat the QD surface facilitate solubility in several common organic solvents. $\mathrm{PbS} / \mathrm{CdS}$ IR QDs are strongly absorptive across the visible wavelength region (as seen in Fig. 1(b)), facilitating efficient light downconversion. In this study, a mixture of QDs and polyisobutylene (PIB) suspended in a mixture of hexane and octane is used as an ink to print the IR emissive layer on top of an indium tin oxide-coated substrate. The role of the polymer matrix is to encapsulate and isolate the QDs from one another (maintaining a higher photoluminescence quantum yield as compared to a film of QDs alone). Additionally, the viscosity of the QD/PIB ink can be adjusted to maintain a high degree of pattern fidelity during inkjet printing [10], [11], enabling printing of side-by-side patterns of multiple "color" inks of different sized QDs. Compatibility of the completed device architecture with flexible substrates is shown in Fig. 1(c).

\section{EXPERIMENT}

Flexible displays are fabricated using ITO-coated polyethylene terephthalate substrates (Sheldahl Inc.) which exhibit $\sim 84 \%$ transmission in the wavelength range of $\lambda=900 \mathrm{~nm}$ to $\lambda=1600 \mathrm{~nm}$. Core/shell $\mathrm{PbS} / \mathrm{CdS}$ QDs are synthesized 


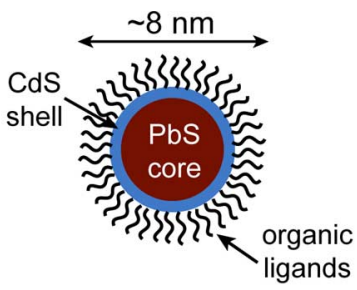

(a)



(b)

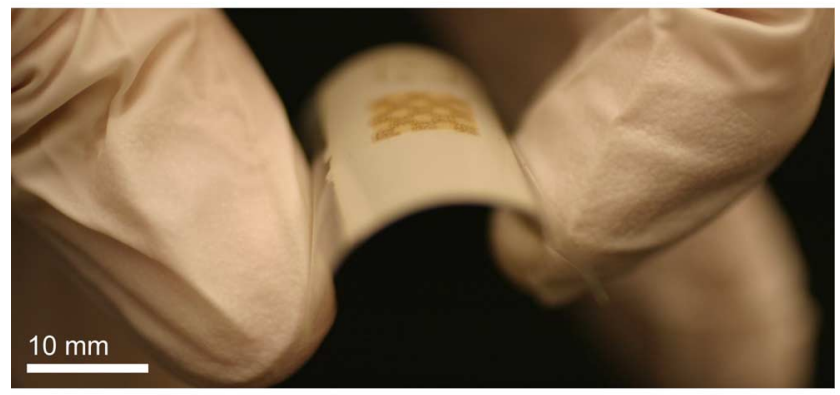

(c)

Fig. 1. (a) Cross-sectional schematic of an individual core/shell $\mathrm{PbS} / \mathrm{CdS}$ QD. (b) Photograph of a chloroform solution of PbS/CdS QDs $(\lambda=1550 \mathrm{~nm}$ peak emission wavelength) under ambient illumination. (c) A completed infrared (IR) display bent to a radius of $\sim 10 \mathrm{~mm}$ to highlight the flexibility of the device fabricated on a plastic substrate.

in-house, following the procedure of Pietryga et al. [12]. A series of QDs with three different peak emission wavelengths is used to prepare three ink solutions by mixing the QDs with a stock solution of $10 \mathrm{mg} \mathrm{mL}^{-1}$ PIB in 9:1 (by volume) hexane:octane, with approximately $400000 \mathrm{~g} \mathrm{~mol}^{-1}$ molecular weight PIB (Acros). Following inkjet printing of the IR emissive layer using a Hewlett Packard Thermal Inkjet Pico-fluidic dispensing System (TIPS), the phosphor layer is formed on top of the printed pattern via doctor blade deposition using a disposable mask. Typical layer thicknesses for the printed IR emissive layer and the phosphor layer are $\sim 1 \mu \mathrm{m}$ and $\sim 500 \mu \mathrm{m}$, respectively.

The blue-emitting phosphor layer used in this study (peak emission wavelength, $\lambda=460 \mathrm{~nm}$ ) is a copper-doped $\mathrm{ZnS}$ powder phosphor (Type 813) dispersed in an insulating binder (Osram-Sylvania). Following doctor blade deposition, the phosphor layer is dried at $50{ }^{\circ} \mathrm{C}$ for 2 hours, after which no visible degradation of the IR emissive layer is observed. The top electrode is then formed by adhering a piece of conductive tape (3M) to the top surface of the phosphor film. Overlap between the top electrode and the bottom ITO layer defines the active device area. To generate electroluminescence of the phosphor layer we apply a $31 \mathrm{kHz}, \sim 350 \mathrm{~V}_{\mathrm{RMS}} \mathrm{AC}$ voltage signal across the electrodes using a JKL inverter (Model BXA-24529) and a 14-V DC power supply. IR emission spectra are measured using a liquid nitrogen-cooled linear InGaAs array detector in conjunction with a monochromator (Princeton Instruments). A colored glass filter is placed in front of the monochromator to block any remaining blue phosphor emission.

\section{RESULTS AND DISCUSSION}

Fig. 2(a) shows a schematic cross-section of the downconversion display. Note that in addition to the structure sketched in Fig. 2(a), we also fabricated displays with the IR emissive layer printed on the bottom (non-ITO coated) side of the substrate. However, this arrangement is less preferable because there is a substantial reduction in the amount of blue phosphor electroluminescence that reaches the IR emissive layer due to waveguiding within the substrate, and the emissive layer is no longer encapsulated between the substrate and the phosphor layer. In order to demonstrate the feasibility of a multicolor IR display, we print side-by-side pixels of two different "color" IR inks, as seen in Fig. 2(b). In order to obtain emission spectra of different peak wavelengths, the size of the $\mathrm{PbS}$ core is varied slightly during colloidal synthesis [6], [13]. PbS/CdS QDs absorb strongly in the visible wavelength region, as seen in Fig. 2(c). Fig. 2(d) shows the normalized emission spectra (driven by blue phosphor electroluminescence) of printed QD/PIB composite layers with three different sizes of QDs used as IR emitters. Peak emission wavelengths (i, ii, iii) of $\lambda=1100 \mathrm{~nm}, \lambda=1350 \mathrm{~nm}$, and $\lambda=1550 \mathrm{~nm}$ are observed. The spectrum of emitter (iii) appears artificially narrow on the higher wavelength side due to the cut-off of the InGaAs detector responsivity at $\lambda=1600 \mathrm{~nm}$. No significant decrease in emission intensity was observed over several hours of periodic display operation.

In order to image the operation of our IR downconversion displays, false-color infrared imaging of operating devices is performed using a cooled InGaAs 2D array camera (XenICs NV) as shown in Fig. 3. Fig. 3(a) (grayscale) shows a display (as viewed through the transparent substrate) with dimensions $\sim 25 \mathrm{~mm} \times 25 \mathrm{~mm}$ supported vertically by a metal clip that is seen in black at the bottom of the image. The two highlighted rectangular regions indicate an area with a printed QD/PIB layer (region " $\mathrm{A}$ ") and an area without the printed IR emitter layer (region "B"). Each rectangular region has its own independently addressable top electrode on the back of the display, while a common phosphor layer is used for both. In Fig. 3(a), no voltage is applied to either device; therefore, the image is made possible by reflected visible broadband light illuminating the substrate. Fig. 3(b) shows the same substrate upon application of a high voltage AC signal to the electrode behind region " $A$ " (with a QD/PIB layer that was printed using solution ii). Strong IR emission from the QDs is clearly visible. In order to demonstrate that blackbody radiation (from device heating) is not responsible for a large IR signal, we image the substrate once more, applying the same AC voltage signal this time to the phosphor-only region "B" [see Fig. 3(c)]. While a small false-color change is observed in the electrified phosphor-only region (either due to device heating or a small response of the InGaAs detector to the blue phosphor emission), it is minimal compared to that seen due to the QD emission.

Key advantages of the IR downconversion display architecture presented here include simple fabrication, the possibility for side-by-side multicolor IR pixel formation, and voltage-driven operation. It is important to note that these displays were fabricated entirely under ambient laboratory conditions, with a single low temperature $\left(50^{\circ} \mathrm{C}\right)$ drying step. Furthermore, in contrast with thin film deposition techniques such as thermal evaporation or spin-coating where material losses can be high, by inkjet printing the emissive layer, very little of the active IR material (QDs) is wasted. Additionally, all fabrication steps are compatible with large area, flexible substrates. By controlling the 


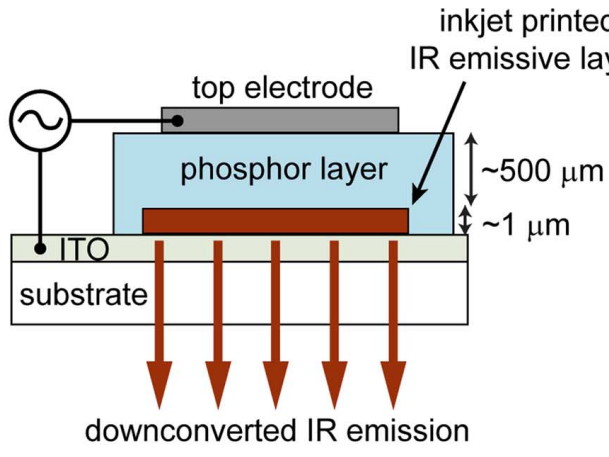

(a)

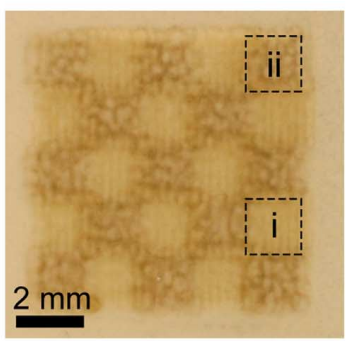

(b)

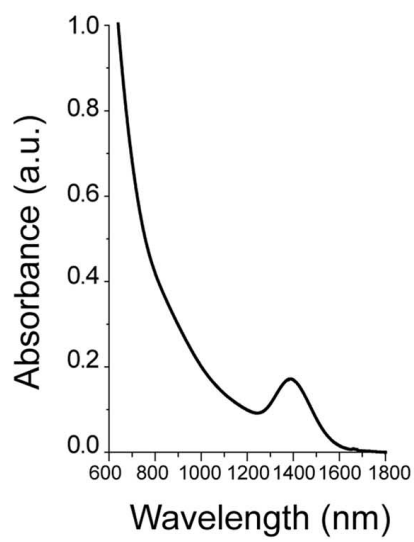

(c)

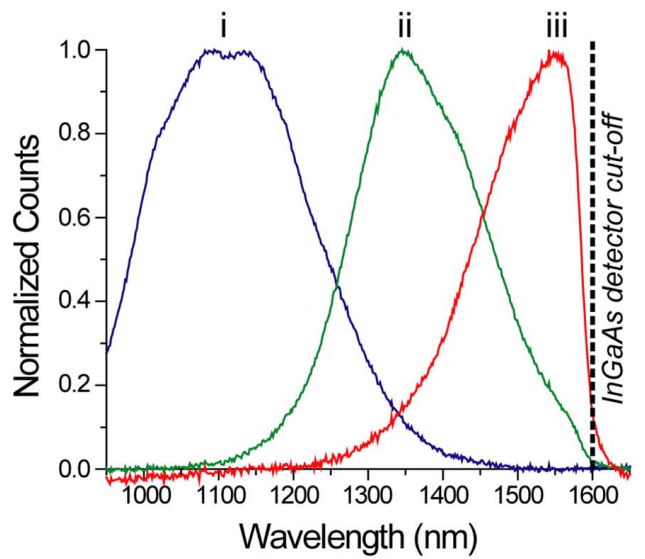

(d)

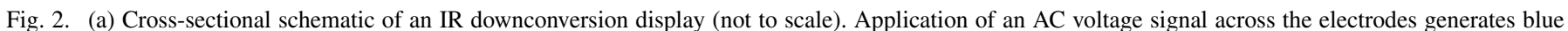

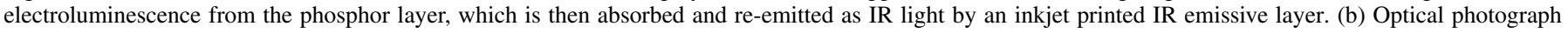

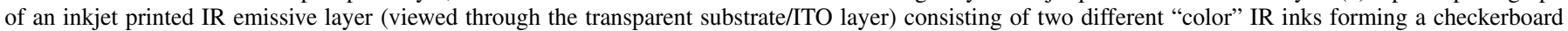

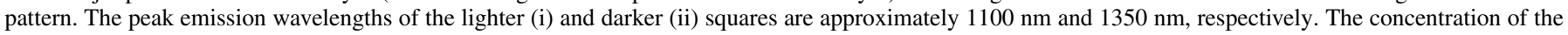

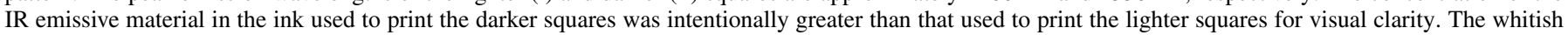

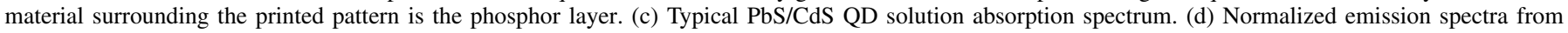

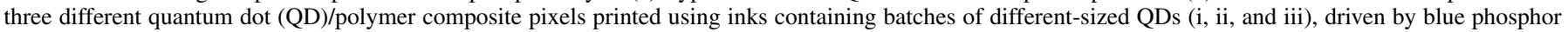

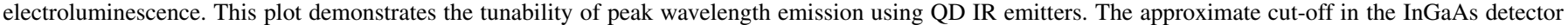
responsivity is shown by a dashed vertical line.

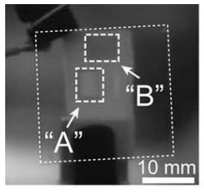

(a)

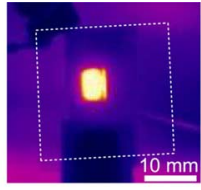

(b)

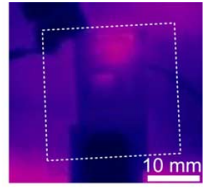

(c)
Fig. 3. Series of false color images of an IR display captured by an InGaAs 2D array camera. Neither the 1 in. $\times 1$ in. glass substrate (outlined by the thin dotted line) nor the camera were moved between subsequent images. The two darker-colored objects common to each image are a metal support clip (bottom) and a metal binder clip used to facilitate electrical connection to the ITO electrode (upper left). (a) Grayscale image of a display containing two distinct pixel areas, one containing a printed QD/polymer IR emissive layer (region "A"), and the other consisting of no IR emissive layer (region "B"). Both areas share a common phosphor layer, and no voltage is supplied to either pixel in this image. (b) Application of a $31 \mathrm{kHz}, \sim 350 \mathrm{~V}_{\mathrm{RMS}}$ AC voltage signal to region "A" shows the strong IR emission of the core/shell PbS/CdS QDs. The emission spectrum of this pixel was peaked at $\lambda=1350 \mathrm{~nm}$. (c) Application of the same AC voltage signal to region "B" (consisting of only the phosphor layer).

size and chemical composition of the QDs during colloidal synthesis, the peak emission wavelength can be tuned across the near IR range. Employing different size QD-based inks, inkjet printing facilitates the definition of side-by-side pixels of dif- ferent IR “colors." This may prove to be attractive for secure information display, since the different peak wavelength-emitting IR pixels could, potentially, all appear as the same color (black or dark brown) to the human eye. In addition, by employing the AC voltage-driven electroluminescence of a relatively thick phosphor layer to optically excite the IR emissive material, we avoid the substantial challenge of developing a current-driven diode architecture.

The efficiency of the IR displays described in this report is given by the product of the blue powder phosphor electroluminescence and the photoluminescence quantum yield (QY) of the $\mathrm{PbS} / \mathrm{CdS}$ QDs in the printed emissive layer (assuming 100\% of the blue light is absorbed). The QY of the as-synthesized QDs (in solution) was measured to be approximately 55\% using an integrating sphere with a germanium photodetector. Upon mixing the QDs into the PIB solution, no change in QY was observed. Due to a partial degree of QD aggregation and energy transfer in the printed composite films, however, we expect the QY of printed QDs in PIB to be somewhat reduced, based on similar observations with visibly-emitting QD composites [10], [14]. Given that the efficiency of the phosphor is on the order of $5 \mathrm{~lm} \cdot \mathrm{W}^{-1}[15]$, even if one assumes a QY of $1 \%$ (a low es- 
timate) for the QDs in the printed composite film, we estimate a time-averaged quantum efficiency of approximately $0.5 \%$ for our AC-driven displays, which is comparable to the efficiency of existing LED structures. Improving the QY of IR-emitting QDs in printed composite films is currently being explored.

\section{CONCLUSION}

In this report, we demonstrate an $\mathrm{AC}$ voltage-driven, multicolor IR display architecture employing an inkjet printed $\mathrm{QD} /$ polymer composite film and an electroluminescent powder phosphor layer. Blue photons emitted by the phosphor are absorbed by core/shell $\mathrm{PbS} / \mathrm{CdS}$ QDs and subsequently re-emitted at well-defined IR wavelengths. This technology is amenable to the fabrication of flexible, large area IR-emissive displays. Using inkjet printing to define side-by-side pixels of composite films containing QDs of different colors (peak emission wavelengths), a multicolor IR display can be realized.

\section{ACKNOWLEDGMENT}

The authors thank L. DeBrouckere at XenICs NV for the use of the InGaAs 2D array camera, as well as G. Su and G. Nair for their valuable laboratory assistance.

\section{REFERENCES}

[1] L. Han, F. Song, S. Q. Chen, C. G. Zou, X. C. Yu, J. G. Tian, J. Xu, $\mathrm{X} . \mathrm{Xu}$, and $\mathrm{G}$. Zhao, "Intense upconversion and infrared emissions in $\mathrm{Er}^{3+}-\mathrm{Yb}^{3+}$ codoped $\mathrm{Lu}_{2} \mathrm{SiO}_{5}$ and $\left(\mathrm{Lu}_{0.5} \mathrm{Gd}_{0.5}\right)_{2} \mathrm{SiO}_{5}$ crystals," Appl. Phys. Lett., vol. 93, pp. 011110-1-011110-3, 2008.

[2] G. Qian, Z. Zhong, M. Luo, D. Yu, Z. Zhang, Z. Y. Wang, and D. Ma, "Simple and efficient near-infrared organic chromophores for lightemitting diodes with single electroluminescent emission above 1000 nm," Adv. Mater., vol. 21, pp. 111-116, 2009.

[3] Y. Yang, R. T. Farley, T. T. Steckler, S. H. Eom, J. R. Reynolds, K. S. Schanze, and J. Xue, "Near infrared organic light-emitting devices based on donor-acceptor-donor oligomers," Appl. Phys. Lett., vol. 93, pp. 163305-1-163305-3, 2008.

[4] Y. Xuan, G. Qian, Z. Wang, and D. Ma, "Near-infrared polymer lightemitting diodes based on infrared dye doped poly $(N$-vinylcarbazole) film," Thin Solid Films, vol. 516, pp. 7891-7893, 2008.

[5] N. Tessler, V. Medvedev, M. Kazes, S. Kan, and U. Banin, "Efficient near-infrared polymer nanocrystal light-emitting diodes," Science, vol. 295, pp. 1506-1508, 2002.

[6] J. S. Steckel, S. Coe-Sullivan, V. Bulović, and M. G. Bawendi, "1.3 $\mu \mathrm{m}$ to $1.55 \mu \mathrm{m}$ tunable electroluminescence from $\mathrm{PbSe}$ quantum dots embedded within an organic device," Adv. Mater., vol. 15, pp. 1862-1866, 2003.

[7] J. M. Caruge, J. E. Halpert, V. Wood, V. Bulović, and M. G. Bawendi, "Colloidal quantum-dot light-emitting diodes with metal-oxide charge transport layers," Nature Photon., vol. 2, pp. 247-250, 2008.

[8] P. O. Anikeeva, C. F. Madigan, J. E. Halpert, M. G. Bawendi, and V. Bulović, "Electronic and excitonic processes in light-emitting devices based on organic materials and colloidal quantum dots," Phys. Rev. B, vol. 78, pp. 085434-1-085434-8, 2008.

[9] Y. A. Ono, Electrolumines. Displays. Singapore: World Scientific, 1995.

[10] V. Wood, M. J. Panzer, J. Chen, M. S. Bradley, J. E. Halpert, M. G. Bawendi, and V. Bulović, "Inkjet printed quantum dot-polymer composites for full color AC-driven displays," Adv. Mater., vol. 21, pp. 2151-2155, 2009.

[11] H. M. Haverinen, R. A. Myllylä, and G. E. Jabbour, "Inkjet printing of light emitting quantum dots," Appl. Phys. Lett., vol. 94, pp. 0731081-073108-3, 2009.

[12] J. M. Pietryga, D. J. Werder, D. J. Williams, J. L. Casson, R. D. Schaller, V. I. Klimov, and J. A. Hollingsworth, "Utilizing the lability of lead selenide to produce heterostructured nanocrystals with bright, stable infrared emission," J. Amer. Chem. Soc., vol. 130, pp. 4879-4885, 2008.
[13] C. B. Murray, D. J. Norris, and M. G. Bawendi, "Synthesis and characterization of nearly monodisperse $\mathrm{CdE}(\mathrm{E}=\mathrm{S}, \mathrm{Se}, \mathrm{Te})$ semiconductor nanocrystallites," J. Amer. Chem. Soc., vol. 115, pp. 8706-8715, 1993.

[14] J. Lee, V. C. Sundar, J. R. Heine, M. G. Bawendi, and K. F. Jensen, "Full color emission from II-VI semiconductor quantum dot-polymer composites," Adv. Mater., vol. 12, pp. 1102-1105, 2000.

[15] K. H. Butler and J. F. Waymouth, "Electroluminescence of zinc sulfide phosphors," Brit. J. Appl. Phys., vol. 6, pp. S33-S38, 1955.

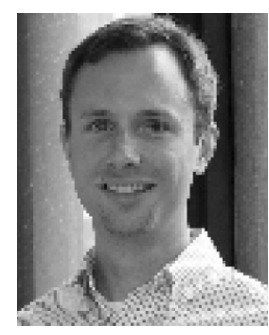

Matthew J. Panzer received the B.Ch.E. degree from the University of Delaware, Newark, in 2002, and the Ph.D. degree in chemical engineering from the University of Minnesota, Minneapolis, in 2007.

Since 2007, he has been with the Laboratory of Organic Optics and Electronics, Massachusetts Institute of Technology, Cambridge, in the area of quantum dot-based optoelectronic devices.

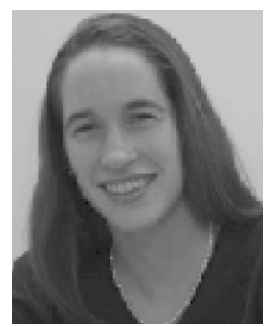

Vanessa Wood received the B.S. degree in applied physics from Yale College, New Haven, CT, in 2005 , and the M.S. degree in electrical engineering from the Massachusetts Institute of Technology, Cambridge, in 2007. She is currently working toward the Ph.D. degree in the Department of Electrical Engineering and Computer Science at the Massachusetts Institute of Technology, working in the area of quantum dotbased optoelectronics.

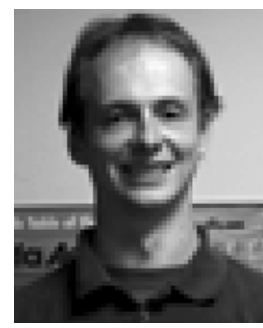

Scott M. Geyer received the B.S. degree in physical chemistry and the B.A. degree in physics from the University of Virginia, Charlottesville, in 2005 . He is currently working toward the Ph.D. degree in the Chemistry Department at the Massachusetts Institute of Technology, Cambridge, MA.

His research is on quantum dot-based optoelectronic devices.

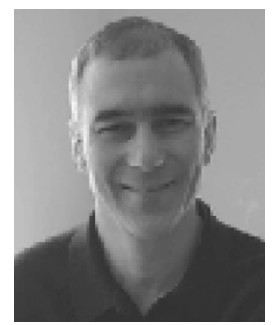

Moungi G. Bawendi received the A.B. degree in chemistry from Harvard University, Cambridge, in 1982, the A.M. degree from Harvard University, Cambridge, in 1983, and the Ph.D. degree from the University of Chicago, Chicago, IL, in 1988.

$\mathrm{He}$ is currently the Lester Wolfe Professor of chemistry at the Massachusetts Institute of Technology, Cambridge, MA.

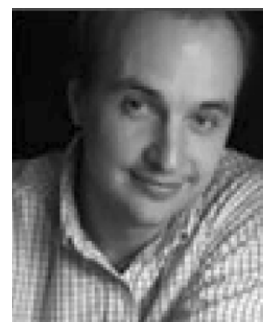

Vladimir Bulović received the B.S.E. degree in electrical engineering from Princeton University, Princeton, in 1991, the M.S. degree from Columbia University, New York, in 1993, and the Ph.D. degree from Princeton University in 1998.

$\mathrm{He}$ is currently an Associate Professor of electrical engineering at the Massachusetts Institute of Technology, Cambridge, MA. 